\title{
EMPODERAMIENTO DE LA CULTURA DE RECICLAJE ORGANICO PARA EL CULTIVO DE ALIMENTOS SALUDABLES EN FAMILIAS LOCALIDAD DE TARMA 2013
}

\author{
Empowerment of the culture of recycling for organic farming families healthy \\ food in town of Tarma 2013
}

\begin{abstract}
Miriam Zulema Espinoza Véliz, ${ }^{1, a, f}$, Marina Luz Elias Moreno ${ }^{2, b, g}$, Rosa Clara Orihuela Espinoza ${ }^{1, a}$ Giovanna Betsabe Enríquez Gonzales ${ }^{3, c, h}$., Oscar Abel Figueroa Mejía, ${ }^{4, d}$, Nelly Marleni Hinostroza Robles ${ }^{1, a, i}$ César Iván ${ }^{i}$ Rojas Jara ${ }^{a, j}$, Elisabet Sara Santos López ${ }^{3, d, k}$

${ }^{1}$ Escuela de Enfermería Universidad Nacional Daniel Alcides Carrión, Filial Tarma. Junín, Perú.

${ }^{2}$ Facultad de Ciencias de la Salud. Universidad Nacional Daniel Alcides Carrión, Sede Central Cerro de Pasco. Pasco, Perú

${ }^{3}$ Escuela de Obstetricia Universidad Nacional Daniel Alcides Carrión Filial Tarma. Junín, Perú

${ }^{4}$ Facultad de Educación. Universidad Nacional Daniel Alcidez Carrión - Sede Central Cerro de Pasco. Pasco, Perú.

${ }^{a}$ Lic. en Enfermería, ${ }^{b}$ Lic. En Estadística, ${ }^{c}$ Lic. en Obstetricia, ${ }^{d}$ Lic. en Educación, ${ }^{e}$ Lic. en Sociología, ${ }^{f}$ Mg. En Investigación y

Tecnología Educativa, ${ }^{g} \mathrm{Mg}$. En Estadística, ${ }^{h} \mathrm{Mg}$. en Salud Pública y Comunitaria, ${ }^{i} \mathrm{Mg}$. en Enfermería, ${ }^{J}$ Doctor en Ciencias de la Salud,

${ }^{k}$ Doctor en Ciencias de la Educación.
\end{abstract}

\section{RESUMEN}

Todos generamos basura, cada ser humano en promedio en el mundo produce de 800 gramos a 1200 gramos de basura diaria, en los hogares se han instaurado ciertas costumbres como: no se separa la basura para eliminarla, utilizar muchos productos descartables, generar residuos orgánicos y no saber reusarlos, claro está, que este es un problema de cultura, de educación, de falta de conciencia ecológica, y que simplemente nos hemos dedicado a contaminar nuestro entorno de vida. Objetivo: Determinar el empoderamiento la cultura de reciclaje orgánico para el cultivo de alimentos saludables en familias localidad de Tarma 2013, se buscó contrastar la hipótesis de que el empoderamiento de la cultura de reciclaje orgánico favorece a un enfoque óptimo para el cultivo de alimentos saludables en familias de la localidad de Tarma. Material y métodos: Investigación cuasi experimental con diseño de un grupo con pre y post test, la muestra la constituyeron 25 familias, se tomó en cuenta los instrumentos de cuestionario y guía de observación antes y después y cuatro módulos para la aplicación de las intervenciones. Resultados: La cultura de reciclaje orgánico para producir el compost casero al inicio de la investigación se ve reflejada mayoritariamente a través del acceso a la información sobre todo en familias de tipo nuclear, la cultura para el cultivo de alimentos saludables es deficiente al inicio de la investigación sobre todo en las familias de tipo monoparental y nuclear, el enfoque de empoderamiento de la cultura de reciclaje orgánico para producir compost casero demostró una alta capacidad después de las intervenciones, el enfoque de empoderamiento de cultura para cultivo de alimentos saludables demostró una característica óptima después de las intervenciones. Conclusiones: El empoderamiento de la cultura de reciclaje orgánico para el cultivo de alimentos saludables después de las intervenciones con las familias sujetos a estudio muestra una característica óptima, viéndose ello reflejado con una alta capacidad de empoderamiento, lo que permitió contrastar la hipótesis planteada.

Palabras Clave: Cultura, Reciclaje, Empoderamiento, Compost, Cultivo, Alimentos saludables.

\section{Summary}

All garbage generated every human being in the world on average produces 800 grams to 1200 grams of daily waste, households have established certain customs as to eliminate waste is not separated, use many disposable products, organic waste and generate reuse them not knowing, of course, that this is a problem of culture, education, lack of environmental awareness, and we have just dedicated to pollute our living environment. Objective: To determine the culture of empowerment organic recycling for growing healthy food in town of Tarma 2013 families, we sought 
to test the hypothesis that the empowerment of organic recycling culture favors an optimal approach for growing healthy food families in the town of Tarma. Material and Method: A quasi-experimental research design with one group pre and post test, the sample comprised 25 families, was taken into account instruments Questionnaire and observation before and after four modules for the implementation of interventions. Results: The culture of recycling organic produce home at the beginning of the investigation compost is reflected mainly through access to information especially in nuclear-type families , culture for growing healthy food is poor at the beginning of the research especially in single-parent families and nuclear families, the empowerment approach of the culture of recycling organic produce home compost showed a high capacity after interventions, cultural empowerment approach to growing healthy food showed an optimal feature after interventions. Conclusions: empowering the culture of recycling organic health food crop after interventions with families under study shows an excellent feature, seeing it reflected a high capacity for empowerment, allowing to test the hypothesis.

Keywords: Culture, Recycling, Empowerment, Compost, Crop, Health Food.

\section{INTRODUCCIÓN}

El ser humano ha experimentado en su quehacer en el mundo un desvío a través del poder de la ciencia y la técnica, más no ve las consecuencias de ello, ya que se encapsula sobre sí mismo y se comporta como mero observador ante la destrucción de la humanidad, del medio ambiente y la ecología.

$\mathrm{El}$ tema medioambiental cobra real importancia cuando nos damos cuenta que los recursos naturales no son inagotables. Muchos de los "recursos naturales" una vez utilizados ya no son recuperables, ni se pueden renovar naturalmente. Por lo tanto llegará un momento en que la tierra y los recursos que hay en ella ya no sean suficientes para sostener a la creciente población del mundo.

Formamos una misma realidad compleja diversa y única con la tierra, la humanidad y la tierra forman una única realidad espléndida, reluciente y a la vez frágil y llena de vigor estamos formados por las mismas energías por tanto debemos conectarnos y cuidarlo (1).

La población puede percibir con creciente malestar el incremento de los problemas relacionados con la forma en que es tratada la basura, desde los insuficientes camiones recolectores, hasta el tratamiento no metódico de la misma, hechos que se traducen en problemas relacionados con insalubridad, enfermedades infectocontagiosas, pobreza y marginalidad. Aunque es relativamente fácil cuantificar el número de toneladas de basura que se producen, los datos disponibles revelan la importancia relativa que significa tratar de hallar una solución conjunta a la problemática que representa la basura, esto es, que la cantidad de basura crece, pero los espacios para almacenarla no, la basura acumulada en grandes cantidades puede afectar el aire, el agua de los ríos, el agua de los mantos que utilizamos para consumo humano y el suelo de las tierras de cultivo, entre otros problemas.

En nuestro País, según el INEI, a mediados de la década de 1950 la generación diaria de residuos sólidos bordeaba las 2.280 toneladas, menos del $10 \%$ de lo que se produce hoy, a partir de 1970 como respuesta al cambio de la dinámica social se dio inicio a una sociedad masiva de consumo y se incrementó la generación de residuos sólidos, los Municipios no lograban cubrir la demanda del servicio de limpieza pública, los recicladores eran pocos y actuaban de manera independiente. En la actualidad, se produce aproximadamente 6,000 toneladas de basura al día, es decir, se genera residuos a un ritmo de 4,2 toneladas por minuto y 2,2 millones de toneladas al año (2). 
La educación ambiental es un elemento básico como un proceso dinámico para transmitir información y transformar el conocimiento, se basa en el empoderamiento para la salud de la comunidad donde supone que los individuos actúen colectivamente con el fin de conseguir una mayor influencia y control sobre los determinantes de la salud y la calidad de vida de su comunidad, implica fomentar, y desarrollar habilidades para la autosuficiencia, con un énfasis en el reforzamiento de actitudes, pensamiento y conductas favorables a la conservación de la naturaleza, el entorno urbano y el desarrollo sostenible. Daniel García Torres (2006) sostiene que formar al hombre es prepararlo para solucionar los problemas que se le presente, formar convicciones que le permita prevenir $y$ solucionar los principales problemas del medio ambiente en el espacio en que se desarrolla, con ello se logra una cultura integral que permita un cambio de conducta frente a su propio desarrollo económico y social lo que ayudaría a cambiar drásticamente la orientación autodestructora de su propia actividad sobre la naturaleza (3).

La educación siempre empieza por casa, los más pequeños deben recibir estímulos y el ejemplo de una conducta respetuosa con el ambiente. En el hogar se forman los hábitos cotidianos de consumo responsable de agua y energía, de reciclaje de residuos y de conductas éticas y meditadas. Las pequeñas pero importantes decisiones que se toman a diario, así como los patrones de consumo, pueden contribuir a aliviar los problemas del planeta, no es complicado tener una conducta de cuidado para con la naturaleza y por consiguiente con nosotros mismos, adquiriendo una "Cultura de Separar la Basura", es el paso número uno que se debe aplicar, para que funcione el cuidado del medio ambiente desde el hogar.

Debemos considerar la necesidad de adoptar nuevos conceptos de riqueza y prosperidad, lo cual no solo permitirá alcanzar mayores estándares de vida mediante el cambio de los estilos de vida, sino también nos hará menos dependientes de los recursos finitos de la Tierra y nos colocará en armonía con las capacidades de ésta (4).

En la presente investigación ¿Como será el empoderamiento de la cultura de reciclaje orgánico para el cultivo de alimentos saludables en familias de la localidad de Tarma 2013?, se buscó que en el contexto familiar se forme la conciencia ciudadana y cultura ecológica para el cuidado del medio ambiente, siguiendo el método científico positivista de cohorte experimental, en busca de dar soluciones tecnológicas a pequeña escala, con el reciclaje de residuos orgánicos que a diario se generan en el hogar y que son eliminados a la basura mezclados con otros residuos, en lugar de ello convertirlos en compost casero y con ello cultivar alimentos que estén a su alcance y contengan gran valor nutritivo, para ser revertidos al cuidado de su salud. Entorno a la educación para la salud y cultura ambiental, en busca de crear Estilos de Vida Saludable. El estudio fue realizado en las familias de la localidad de Tarma, tuvo una duración de Enero a Diciembre del 2013.

El objetivo de este trabajo fue, determinar el empoderamiento, la cultura de reciclaje orgánico para el cultivo de alimentos saludables en familias localidad de Tarma 2013.

\section{MATERIALES Y METODOS}

El Estudio fue experimental, con diseño cuasi experimental de grupo único no equivalentes, con pre y post test, la muestra estuvo compuesta por 25 familias, se hizo uso de una guía de observación para hacer el seguimiento de las intervenciones, se recopiló datos acerca de conocimientos sobre cultivo de alimentos saludables y cultura de reciclaje orgánico, mediante el cuestionario, se hizo uso de cuatro módulos que condujeron el protocolo del estudio. Para el análisis estadístico de los cuadros con datos cualitativos se hizo uso de la prueba no para métrica de la Ji CUADRADA (5). 
RESULTADOS.

TABLA No 01. CULTURA DE RECICLAJE ORGÁNICO PARA PRODUCIR EL COMPOST CASERO SEGÚN TIPO DE FAMILIAS TEST ANTERIOR- LOCALIDAD DE TARMA 2013

\begin{tabular}{|c|c|c|c|c|c|c|c|c|}
\hline \multirow{2}{*}{$\begin{array}{c}\text { TIPO DE FAMILIA } \\
\text { CULTURA DE RECICLAJE } \\
\text { ORGÁNICO }\end{array}$} & \multicolumn{2}{|c|}{ Nuclear } & \multicolumn{2}{|c|}{ Extensa } & \multicolumn{2}{|c|}{ Monoparental } & \multicolumn{2}{|c|}{ TOTAL } \\
\hline & $\mathrm{N}^{0}$ & $\%$ & $\mathrm{~N}^{0}$ & $\%$ & $\mathrm{~N}^{0}$ & $\%$ & $\mathrm{~N}^{0}$ & $\%$ \\
\hline Acceso a la información & 5 & 20 & 1 & 4 & 2 & 8 & 8 & 32 \\
\hline Inclusión y participación & 2 & 8 & 1 & 4 & 1 & 4 & 4 & 16 \\
\hline Responsabilidad & 2 & 8 & 3 & 12 & 2 & 8 & 7 & 28 \\
\hline Capacidad de organización & 3 & 12 & 1 & 4 & 2 & 8 & 6 & 24 \\
\hline TOTAL & 12 & 48 & 6 & 24 & 7 & 28 & 25 & 100 \\
\hline
\end{tabular}

FUENTE: Cuestionario aplicado por los investigadores

En la Tabla $N^{\circ} 01$, se demuestra la cultura de reciclaje orgánico que tienen inicialmente las familias sujetos a estudio para producir el compost casero; de los cuales en un $32 \%$ (08 familias), se ve reflejada mayoritariamente a través del acceso a la información sobre todo en las familias de tipo nuclear con un $20 \%$ (05 familias), un $28 \%$ (07 familias) demuestran responsabilidad, viéndose reflejada mayoritariamente con un 12\% (03 familias) en las familias de tipo extensa, un 24\% (06 familias) demuestra capacidad de organización siendo ello de mayor preponderancia en las familias de tipo nuclear con un $12 \%$ (03 familias) y solo un $16 \%$ (4 familias) demuestra inclusión y participación, reflejándose más en las familias de tipo nuclear con un 8\% (02 familias). No obstante la comprobación estadística, obliga a aceptar la hipótesis nula y rechazar la hipótesis alterna, teniendo en cuenta un 95\% de confiabilidad, permitiendo concluir que no hay relación significativa entre las variables, pero si resaltar que: la cultura de reciclaje orgánico para producir el compost casero se ve reflejada mayoritariamente a través del acceso a la información sobre todo en las familias de tipo nuclear.

TABLA N 02. CULTURA PARA EL CULTIVO DE ALIMENTOS SALUDABLES QUE TIENEN LAS FAMILIAS DE LA LOCALIDAD DE TARMA 2013

\begin{tabular}{|c|c|c|c|c|c|c|c|c|}
\hline \multirow{2}{*}{$\begin{array}{c}\text { TIPO DE FAMILIA } \\
\text { CULTURA PARA EL CULTIVO DE } \\
\text { ALIMENTOS SALUDABLES }\end{array}$} & \multicolumn{2}{|c|}{ Nuclear } & \multicolumn{2}{|c|}{ Extensa } & \multicolumn{2}{|c|}{ Monoparental } & \multicolumn{2}{|c|}{ TOTAL } \\
\hline & $\mathrm{N}^{0}$ & $\%$ & $\mathrm{~N}^{0}$ & $\%$ & $\mathrm{~N}^{0}$ & $\%$ & $\mathrm{~N}^{0}$ & $\%$ \\
\hline OPTIMO & 3 & 12 & 2 & 8 & 1 & 4 & 6 & 24 \\
\hline REGULAR & 5 & 20 & 1 & 4 & 2 & 8 & 8 & 32 \\
\hline DEFICIENTE & 4 & 16 & 3 & 12 & 4 & 16 & 11 & 44 \\
\hline TOTAL & 12 & 48 & 6 & 24 & 7 & 28 & 25 & 100 \\
\hline
\end{tabular}

FUENTE: Cuestionario aplicado por los investigadores

En la Tabla $\mathrm{N}^{\circ} 02$, se demuestra la cultura para el cultivo a alimentos saludables que tienen inicialmente las familias sujetos a estudio; de los cuales en un 44\% (11 familias), reflejan una característica deficiente y esto mayoritariamente se da en las familias de tipo monoparental y nuclear con un $16 \%$ (04 familias) respectivamente, un 32\% (08 familias) demuestran una característica regular, viéndose reflejada mayoritariamente con un 20\% (05 familias) en las familias de tipo nuclear y un 24\% (06 familias) demuestra capacidad optima siendo ello de mayor preponderancia en las familias de tipo nuclear con un $12 \%$ (03 familias). Mediante la comprobación estadística se acepta la hipótesis nula y rechaza la hipótesis alterna con un $95 \%$ de confiabilidad, determinando que no hay relación significativa entre la cultura para el cultivo de alimentos saludables y el tipo de familia de la localidad de Tarma, no obstante se puede resaltar que: la cultura para el cultivo de alimentos saludables es deficiente al inicio de la investigación sobre 
Empoderamiento de la Cultura de reciclaje orgánico

todo en las familias de tipo monoparental y nuclear.

TABLA $\mathbf{N}^{0}$ 03. ENFOQUE DE EMPODERAMIENTO DE LA CULTURA DE RECICLAJE ORGÁNICO PARA PRODUCIR COMPOST CASERO EN LOS HOGARES DE LAS FAMILIAS DE LA LOCALIDAD DE TARMA 2013

\begin{tabular}{lccccccccc}
\hline \multicolumn{1}{c}{ TIPO DE FAMILIA } & \multicolumn{2}{c}{ Nuclear } & \multicolumn{2}{c}{ Extensa } & \multicolumn{2}{c}{ Monoparental } & TOTAL \\
ENFOQUE DE EMPODERAMIENTO DE LA & & & & & & & \\
\cline { 2 - 10 } CULTURA DE RECICLAJE ORGÁNICO & $\mathrm{N}^{0}$ & $\%$ & $\mathrm{~N}^{0}$ & $\%$ & $\mathrm{~N}^{0}$ & $\%$ & $\mathrm{~N}^{0}$ & $\%$ \\
\hline BAJA CAPACIDAD & 1 & 4 & 1 & 4 & 1 & 4 & 3 & 12 \\
EN PROCESO & 1 & 4 & 1 & 4 & 1 & 4 & 3 & 12 \\
ALTA CAPACIDAD & 10 & 40 & 4 & 16 & 5 & 20 & 19 & 76 \\
TOTAL & 12 & 48 & 6 & 24 & 7 & 28 & 25 & 100 \\
\hline
\end{tabular}

FUENTE: módulos de intervenciones con las familias

En la Tabla $\mathrm{N}^{\circ} 3$ se demuestra el enfoque de empoderamiento de la cultura de reciclaje orgánico para producir compost casero después de las intervenciones con las familias sujetos a estudio; de los cuales un $76 \%$ (19 familias), muestran una alta capacidad de empoderamiento viéndose ello reflejado mayoritariamente en las familias de tipo nuclear con un $40 \%$ (10 familias), un $12 \%$ (03 familias) demuestran un empoderamiento en proceso en los tres tipos de familias y de igual manera se puede resaltar que existe baja capacidad de empoderamiento 12\% (03 familias) en los tres tipos de familias. Mediante la comprobación estadística se arribó que no hay relación significativa entre enfoque de empoderamiento de la cultura de reciclaje orgánico para producir compost casero y el tipo de familias de la localidad de Tarma, pero si resaltar que: el enfoque de empoderamiento de la cultura de reciclaje orgánico para producir compost casero demostró una alta capacidad después de las intervenciones.

TABLA $N^{0}$ 4. ENFOQUE DE EMPODERAMIENTO DE CULTURA PARA CULTIVO DE ALIMENTOS SALUDABLES EN LOS HOGARES DE LAS FAMILIAS DE LA LOCALIDAD DE TARMA 2013

\begin{tabular}{|c|c|c|c|c|c|c|c|c|}
\hline \multirow{2}{*}{$\begin{array}{c}\text { TIPO DE FAMILIA } \\
\text { ENFOQUE DE EMPODERAMIENTO DE } \\
\text { CULTURA PARA CULTIVO DE ALIMENTOS } \\
\text { SALUDABLES }\end{array}$} & \multicolumn{2}{|c|}{ Nuclear } & \multicolumn{2}{|c|}{ Extensa } & \multicolumn{2}{|c|}{ Monoparental } & \multicolumn{2}{|c|}{ TOTAL } \\
\hline & $\mathrm{N}^{0}$ & $\%$ & $\mathrm{~N}^{0}$ & $\%$ & $\mathrm{~N}^{0}$ & $\%$ & $\mathrm{~N}^{0}$ & $\%$ \\
\hline OPTIMO & 11 & 44 & 5 & 24 & 6 & 24 & 22 & 88 \\
\hline REGULAR & 1 & 4 & 1 & 4 & 1 & 4 & 3 & 12 \\
\hline DEFICIENTE & -- & -- & -- & -- & -- & -- & -- & -- \\
\hline TOTAL & 12 & 48 & 6 & 24 & 7 & 28 & 25 & 100 \\
\hline
\end{tabular}

FUENTE: Modulo de Intervención con la familia y cuestionario

En la Tabla $N^{\circ} 4$ se demuestra el enfoque de empoderamiento de cultura para cultivo de alimentos saludables después de las intervenciones con las familias sujetos a estudio; de los cuales un $88 \%$ (22 familias), muestran una característica optima viéndose ello reflejado mayoritariamente en las familias de tipo nuclear con un $44 \%$ (11 familias) y un $12 \%$ (03 familias) demuestran un empoderamiento regular en los tres tipos de familias. La comprobación estadística demostró que no hay relación significativa entre enfoque de empoderamiento de cultura para el cultivo de alimentos saludables y el tipo de familias de la localidad de Tarma, cabe resaltar que el enfoque de empoderamiento de cultura para cultivo de alimentos saludables demostró una característica optima después de las intervenciones. 
TABLA N ${ }^{0}$ 5. EMPODERAMIENTO DE LA CULTURA DE RECICLAJE ORGÁNICO PARA EL CULTIVO DE ALIMENTOS SALUDABLES EN FAMILIAS LOCALIDAD DE TARMA 2013

\begin{tabular}{lcccccccc}
\hline $\begin{array}{c}\text { EMPODERAMIENTO DE LA } \\
\text { CULTURA DE RECICLAJE } \\
\text { ORGÁNICO }\end{array}$ & $\begin{array}{c}\text { BAJA } \\
\text { CAPACIDAD }\end{array}$ & $\begin{array}{c}\text { EN } \\
\text { PROCESO }\end{array}$ & \multicolumn{2}{c}{$\begin{array}{c}\text { ALTA } \\
\text { CAPACIDAD }\end{array}$} & TOTAL \\
\cline { 2 - 7 } $\begin{array}{c}\text { ENFOQUE DE CULURA PARA } \\
\text { EL CULTIVO DE ALIMENTOS }\end{array}$ & $\mathrm{N}^{0}$ & $\%$ & $\mathrm{~N}^{0}$ & $\%$ & $\mathrm{~N}^{0}$ & $\%$ & $\mathrm{~N}^{0}$ & $\%$ \\
$\quad$ SALUDABLES & & & & & & & & \\
\hline OPTIMO & 1 & 4 & 2 & 8 & 20 & 80 & 23 & 92 \\
REGULAR & 1 & 4 & 1 & 4 & -- & -- & 2 & 8 \\
DEFICIENTE & -- & -- & -- & -- & -- & -- & -- & -- \\
TOTAL & 2 & 8 & 3 & 12 & 20 & 80 & 25 & 100 \\
\hline
\end{tabular}

FUENTE: Cuestionario

En la Tabla $N^{\circ} 5$ se demuestra el empoderamiento de la cultura de reciclaje orgánico para el cultivo de alimentos saludables después de las intervenciones con las familias sujetos a estudio; de los cuales un 92\% (23 familias), muestran una característica óptima para el cultivo de alimentos saludables, viéndose ello reflejado con una alta capacidad de empoderamiento de la cultura de reciclaje orgánico con un $80 \%$ (20 familias) y solo un $08 \%$ (02 familias) demuestran una regular capacidad para el cultivo de alimentos saludables con una cultura de empoderamiento de reciclaje orgánico en proceso y baja capacidad con un 4\% (1 familia) respectivamente.

Entonces como el estadístico $x^{2}=9,14$ es mayor que el valor crítico 5,99 y este cae en la zona de rechazo se rechaza la hipótesis nula y aceptar la hipótesis alterna, teniendo un $95 \%$ de confiabilidad, por lo que finalmente se puede concluir que, "El empoderamiento de la cultura de reciclaje orgánico favorece a un enfoque óptimo para el cultivo de alimentos saludables en familias de la localidad de Tarma", aceptándose con ello la hipótesis central.

\section{DISCUSIÓN:}

Al enfocar la investigación ¿Cómo será el empoderamiento de la cultura de reciclaje orgánico para el cultivo de alimentos saludables en familias localidad de Tarma 2013?, se buscó desarrollar una nueva cultura de ciudadanía, sustentada en el cuidado del medio ambiente y uso creativo y saludable de los recursos. En nuestra misión de promover estilos de vida saludables.

El dominio de los conceptos es esencial en el entendimiento humano, sin ellos no hay conocimiento ni puede haber aprendizaje y práctica, el hombre debe adquirir una cultura general integral cada vez más amplia que le permita una conducta ambiental (3).

En tal sentido los resultados a los que se arribó en el primer cuadro, demuestra que la cultura de reciclaje orgánico para producir el compost casero al inicio de la investigación se ve reflejada mayoritariamente a través del acceso a la información sobre todo en las familias de tipo nuclear.

Como bien se tiene por conocido en estos últimos tiempos mucho se tocan los tópicos de la ecología, del cuidado del medio ambiente, el rol que tenemos todos los ciudadanos, el cómo y porqué de RECICLAR, en fin se mueve mucha información al respecto, pero no obstante se siguen observando en el cotidiano, la deficiente cultura ecológica y esto porque aún no se puede consolidar la expansión en la libertad de escoger y de actuar, la autoridad y poder del individuo sobre los recursos y las decisiones que afectan a su vida y los comportamientos en adopción de nuevos hábitos y potenciar nuevos valores, esto quiere decir que no hay inclusión, participación, responsabilidad y capacidad de organización que permita a cada ser humano desarrollar un rol determinado para vivir en un ambiente sano y equilibrado ecológicamente, como lo podemos también advertir en la 
investigación realizada por la Central de Medios Mindshare Argentina, "Principales Características del Consumidor Ecológicamente Comprometido LOHAS" realizada en Agosto-Septiembre 2008, donde concluyen que en cuanto al cuidado del medioambiente, el $59 \%$ de los encuestados reconoce que la mejora de la ecología del planeta depende directamente de los hábitos cotidianos, pero no todos han adecuado sus estilos de vida y costumbres a los asuntos ambientales, ya que sólo el $22 \%$ de los consumidores responsables han sacrificado su comodidad y cambiado algunas convicciones en función de colaborar con el cuidado del medio ambiente (6).

El segundo resultado encontrado en la presente investigación está referido a que la cultura para el cultivo de alimentos saludables es deficiente al inicio de la investigación sobre todo en las familias de tipo monoparental y nuclear. Si bien es cierto que para cuidar el medio ambiente, se debe partir por cuidarse uno mismo, pero en el caso actual, se está viviendo la cultura del descuido sobre todo cuando recurrimos al tema alimenticio, son pocas las familias que conocen el cómo realizar el cultivo de alimentos saludables ya que este no es un bien que se pueda donar, sino un proceso dinámico del que la propia gente es protagonista mediante sus propios esfuerzos individuales y colectivos, para alimentarse a través de mejores condiciones apelando a su creatividad y su enfoque de vivir mejor.

Los resultados encontrados en el estudio coinciden con los encontrados por Aguilar M, y García, M. (2009), en la investigación "Las amas de casa reciclan más y tienen una mayor conciencia ecológica que los universitarios", donde refieren que "una mayor concienciación hacia el medio ambiente no siempre lleva a la puesta en marcha de conductas ecológicamente responsables. Muchas personas que se consideran "ecologistas" o manifiestan una actitud favorable al medio ambiente, pero sin embargo, a nivel conductual "el repertorio de conductas ecológicas que realizan es escaso" (7).

Los tres últimos resultados del estudio, reflejan la factibilidad y viabilidad para responder a retos, es decir, el análisis de contexto y el protagonismo de la gente, brindó la forma más adecuada de promover el empoderamiento mediante enfoques participativos: si las conductas se aprenden, se practican y se asimilan en el ser se convierten en una responsabilidad social.

En tal sentido se destaca que el enfoque de empoderamiento de la cultura de reciclaje orgánico para producir compost casero demostró una alta capacidad después de las intervenciones. La alta capacidad está referida a que las familias lograron: autoconfianza, asertividad cultural, autogestión, autosuficiencia y acceso al conocimiento y habilidades para la formación y resolución de problemas.

El enfoque de empoderamiento de cultura para cultivo de alimentos saludables demostró una característica óptima después de las intervenciones. Refiriéndose que las familias manejan un alto conocimiento sobre los tipos de alimentos de la zona, aporte nutricional, sus características para el cultivo, sus aspectos de cuidado y sus efectos en el organismo

Ambos resultados pueden reforzarse también con la investigación realizada por la Central de Medios Mindshare Argentina, "Principales Características del Consumidor Ecológicamente Comprometido LOHAS" donde advierten que: "Respecto al estilo de vida, el $92 \%$ de los casos afirma estar convencido de que la calidad de las relaciones humanas, la justicia social, el desarrollo personal y la vida sustentable son las claves del mundo en el que quieren vivir" (6).

A su vez también se coincide con Varsavsky, A.I. en su estudio Química Verde y Prevención de la Contaminación Industria y Química Argentina 2002, donde concluye que "La llamada química verde o química sustentable tiene como barreras la 
generalización de estrategias culturales que convierten a la educación en un componente fundamental en toda estrategia sustentable" (8).

En el último resultado se encontró que el empoderamiento de la cultura para el cultivo de alimentos saludables después de las intervenciones con las familias sujetos a estudio muestra una característica óptima, viéndose ello reflejado con una alta capacidad de empoderamiento de la cultura de reciclaje orgánico.

Este resultado central es convergente con el estudio realizado por Fernández, D. (2003) "El compostaje de residuos, una mirada a los biosólidos", quien concluye que; "el compostaje permite un proceso de biodegradación oxidativa en condiciones controladas y es una alternativa de reciclado y valorización que permite transformar la fracción orgánica de ciertos residuos en un producto capaz de ser utilizado para diversos fines". (9)

A su vez también se refuerza con lo referido por Aguilar, M. y García M., en su estudio "Las amas de casa reciclan más y tienen una mayor conciencia ecológica que los universitarios", donde refiere en dos de sus conclusiones: "Los factores sociales influencian en la denominada "norma personal" o "norma moral" (el sentimiento de obligación moral, que recoge una parte ética o moral hacia el medio ambiente) que tengamos hacia la conducta, y el segundo, si se proponen programas destinados a hacer conscientes a las personas tanto de las consecuencias de la acción de reciclar como de las de no hacerlo, que permitan generar una determinada "ética" o moralidad en torno a los temas medio ambientales, posiblemente haremos que se produzca un incremento en la frecuencia de esta conducta ecológica". (7)

Se concluye que, "El empoderamiento de la cultura de reciclaje orgánico favorece a un enfoque óptimo para el cultivo de alimentos saludables en familias de la localidad de Tarma".
Esta investigación contribuye a lo explicitado por Jiménez, L. que se han abierto caminos de investigación transdiciplinarios con una perspectiva sistémica, que permita cambios responsables en la población. $Y$ asumir la triple dimensión de la sostenibilidad en un nuevo marco ético; que considera a la sostenibilidad integral como la sumatoria de la sostenibilidad ambiental, sostenibilidad social y la sostenibilidad económica (10).

Asimismo López. V. considera que se deben establecer programadas encaminados a la sustentabilidad con instituciones capaces de generar cambios, para conservar el medio ambiente (11).

\section{CONCLUSIONES}

El $48 \%$ de familias de tipo nuclear adoptan una cultura de reciclaje orgánico para producir el compost casero. El $32 \%$ de las familias lo hacen porque tienen acceso a la información.

La cultura para el cultivo de alimentos saludables es deficiente en un $44 \%$ de familias. El enfoque de empoderamiento de la cultura de reciclaje orgánico para producir compost casero demostró una alta capacidad después de las intervenciones en un $76 \%$ de las familias.

El empoderamiento de la cultura de reciclaje orgánico favorece a un enfoque óptimo para el cultivo de alimentos saludables en un $92 \%$ de las familias. Esto se refleja por que las familias lograron; autoconfianza, asertividad cultural, autogestión, autosuficiencia, acceso al conocimiento y habilidad para la formación de hábitos y resolución de problemas, adoptando estilos de vida saludables.

\section{Correspondencia:}

Nelly Hinostroza Robles.

Correo electrónico: nmhinostroza@outklook.com 


\section{REFERENCIAS BIBLIOGRÁFICAS:}

1. Boff L. Saber Cuidar: Ética del Cuidado Humano - Compasión por la Tierra" Primera Edición, Brasil 1999, p. 68 al 70.

2. INEI. Perú Medio Ambiente. Residuos Sólidos [Fecha de acceso 12 agosto del 2013] URL Disponible en: www.inei.go.pe/media/...Cap.5 pdf.

3. García D. Morosen R. Formación de una conciencia ambiental en el hombre moderno. Un reto para el nuevo milenio. Universidad de Oriente. Cuba 2004

4. CEADU. Consumo Sustentable [Fecha de acceso 20 de agosto del 2013] URL Disponible en: wwwceadu.org.uy/consumo sustentable.

5. Polit y Hungler. "Investigación Científica en Ciencias de la Salud", Sexta Edic. Edit. Interamericana. México 2000. p. 446, 459, 461.
6. Central de Medios Mindshare Argentina. Principales características del consumidor ecológicamente comprometido LOHAS. Agosto-Setiembre 2008

7. Aguilar M., García M. Amas de casa reciclan más y tienen mayor conciencia ecológica que universitarios. Universidad Granada 2009

8. Varsavsky A. Química Verde y Prevención de la contaminación Industria y Química. Argentina 2002

9. Fernández D. El compostaje de residuos. Una mirada de los biosólidos. Industria y Quimica. Argentina 2003

10. Jiménez L. "Desarrollo Sostenible y Economía Ecológica”, segunda edición, México. 2005. p. 65 al 77. 273 al 278.

11. López V. "Sustentabilidad y Desarrollo Sustentable", segunda edición, México 2008. p. 54 y $55,173$.

Recibido: 30/07/2014

Aprobado para publicación: 22/11/2014 